\title{
Co-Culture with Chorionic Villous Mesenchymal Stem Cells Promote Endothelial Cell Proliferation and Angiogenesis via ABCA9-AKT Pathway
}

\section{Yijing Chu}

Department of Obstetrics, the Affiliated Hospital of Qingdao University, Qingdao, China Jianxin Zuo

Department of Obstetrics, the Affiliated Hospital of Qingdao University, Qingdao, China

\section{Yan Zhang}

Department of Obstetrics, the Affiliated Hospital of Qingdao University, Qingdao, China

\section{Guoqiang Gao}

Department of Obstetrics, the Affiliated Hospital of Qingdao University, Qingdao, China

\section{Xiaoyu Hu}

Department of Obstetrics, the Affiliated Hospital of Qingdao University, Qingdao, China

\section{Rendong Han}

Department of Obstetrics, the Affiliated Hospital of Qingdao University, Qingdao, China

\section{Chong Liu}

Department of Obstetrics, the Affiliated Hospital of Qingdao University, Qingdao, China

Huansheng Zhou

Department of Obstetrics, the Affiliated Hospital of Qingdao University, Qingdao, China

Min Li

Department of Obstetrics, the Affiliated Hospital of Qingdao University, Qingdao, China

\section{Wei Peng}

Department of Obstetrics, the Affiliated Hospital of Qingdao University, Qingdao, China

\section{Yan Wang ( $\nabla$ sanwang1986@163.com )}

Affiliated Hospital of Medical College Qingdao University: The Affiliated Hospital of Qingdao University https://orcid.org/0000-0002-6460-9160

\section{Research}

Keywords: CV-MSCs, ABCA9, AKT, HUVEC, Preeclampsia

Posted Date: December 16th, 2020

DOI: https://doi.org/10.21203/rs.3.rs-126796/v1 
License: (c) (i) This work is licensed under a Creative Commons Attribution 4.0 International License. Read Full License 


\section{Abstract}

Objective

It is found that human chorionic villous mesenchymal stem cells (CV-MSCs) are a promising and effective treatment for tissue injury. Trophoblast dysfunction during pregnancies obviously entangles to the pathogenesis of preeclampsia (PE). This work was to make it clear how CV-MSCs regulated vascular endothelial cell function.

\section{Methods}

In this study, we treated HUVEC with CV-MSC CM and RNA-seq analysis was used to understand the changes in HUVEC. We examined the levels of ABCA9 and AKT signaling in HUVEC by immunohistochemistry, western blot and qRT-PCR assays. CCK-8, colony formation and tube formation assays were used to understand the role of ABCA9 in the growth and angiogenesis of HUVEC mediated by CV-MSCs.

Results

The CV-MSCs treatment significantly strengthened enhanced the HUVEC angiogenesis and proliferation. Furthermore, a significant increase of ABCA9 expression and activation of the AKT pathway in CV-MSCs treated HUVEC was observed. Consistent with these findings, $A B C A 9$ overexpression exhibited the same effect of promoting HUVEC proliferation and angiogenesis as induced by CV-MSC CM, also with the accompaniment of activation of AKT signaling. In addition, inhibition of ABCA9 inactivated the AKT signaling in HUVEC, and reduced the proliferation and angiogenesis of HUVEC. Importantly, the elevation of proliferation and angiogenesis induced by ABCA9 overexpression in HUVEC could be attenuated by ABCA9 overexpression.

\section{Conclusion}

Our results suggest that ABCA9-dependent AKT signaling activation mediated by CV-MSCs could promote the proliferation and angiogenesis of HUVECs.

\section{Introduction}

Vascular endothelial cell is a type of epithelium which exists in blood vessels' the interior surface. It referred to various aspects of vascular biology, including blood clotting, angiogenesis and barrier function ${ }^{1,2}$. Therefore, endothelial dysfunction is often regarded as a hallmark for many vascular diseases, including hypertension, atherosclerotic diseases, and preeclampsia ${ }^{3,4}$. Preeclampsia is a pregnancy-specific disorder affecting $2-8 \%$ of all pregnancies ${ }^{5}$. The poorly established placenta produces anti-angiogenetic factors inducing generalized systemic inflammation and endothelial cell dysfunction, resulting in clinical signs of preeclampsia, such as hypertension and proteinuria ${ }^{6,7}$. To date, although the 
potential mechanisms underlying the pathogenesis of preeclampsia remain unclear, endothelial cellular injury has become a major research focus of the pathogenetic processing ${ }^{8}$.

Derived from BM which adhered to formed and plastic fibroblast colonies, mesenchymal stem cells (MSCs) were firstly to be considered as spindle shaped cells, and they are able to turn into cell derivatives of the mesenchymal lineage, such as myocytes, osteocytes, adipocytes and chondrocytes ${ }^{9,10}$. MSCs have been derived from various adult tissues, such as amniotic fluid, and umbilical cord blood, liver, adiposetissue, bone marrow, endometrium, dental pulp, muscle and placenta ${ }^{11}$. Because of their innumerable biological characteristics connected with the ease of their obtention and in vitro expansion, MSCs have played an important role in the cell therapy-based regenerative medicine field ${ }^{12-14}$. With the increasing knowledge about MSC regenerative property, more and more attention to their clinical potential is paid. For example, MSCs significantly reduce cell angiogenesis and proliferation ${ }^{15,16}$. Moreover, MSCs are found to have antimicrobial, antiapoptotic, and immunoregulatory functions ${ }^{17,18}$. The more important thing is, it has been discovered that placental cells play an important part in regulating peroxidation reactions and specific damaged tissue recovery ${ }^{18,19}$. Placental MSCs are shown to have an effect on experimental animal models with cerebral ischemia, spinal cord injury, Parkinson's disease, ischemic brain disease and Alzheimer's disease by taking advantage of cytokines and/or neurotrophic factors secreted from placental MSCs and angiocrine growth factors ${ }^{20-22}$. Whereas, information about the chorionic villous mesenchymal stem cells (CV-MSCs) in vascular endothelial cells in placental umbilical cord circulation, including cellular proliferation, migration, and formation of capillary-like tube structures, is limited. Therefore, the present study used CV-MSCs conditional medium treated with Human Umbilical Vein Endothelial Cells (HUVECs), and then investigate whether and how CV-MSCs would be affected endothelial cells.

Transcriptome analyses manifested considerable upregulation of ABCA9 in HUVEC by CV-MSCs CM. In addition, the expression of ABCA9 affected AKT signalling in HUVEC with corresponding changes of proliferation and tube formation capability. Our results confirmed that CV-MSCs could promote proliferation and tube formation of HUVEC through ABCA9 and AKT regulation.

\section{Materials And Methods}

\section{Patients and samples}

The use of human placentas and all experiments were supported by the Ethical Committee of the Affiliated Hospital of Qingdao University (Shandong, China). Moreover, to participate in the research, all of the volunteers signed the written informed consent. With parental permission, the age-matched placentas from severe preeclampsia after caesarean section $(n=10)$, while placentas were derived from full-term births $(n=10)$. All placentas were gathered through elective cesarean section with induced labor. Besides, placental tissues were collected by the way of getting rid of a vertical plane through the full thickness of an obviously normal and central area, containing not only the maternal but also fetal surfaces. However, tissues with clots or calcification were not used. 


\section{Cell Culture and CV-MSC conditioned medium preparation}

The above-mentioned placentas tissues were was minced and removed blood. Then collagenase $(0.1 \%)$ and trypsin (0.25\%) (type I; Sigma-Aldrich, St. Louis, MO) were made use of to digest the tissues for 30 min. After filtrating through a 100- $\mu$ m nylon filter and centrifugation, the cells were placed on culture plates in a stem cell culture medium (SCCM), and the culture medium consisted of $5 \%$ UltraGRO ${ }^{\text {TM }}$ (Helios, USA) and Stem Cell Basic Medium (Dakewe Biotech Co., Guangzhou, China). Besides, an incubator, with $5 \% \mathrm{CO}_{2}$ and thirty-seven degrees Celsius, was applied to culture the primary cells. In this study, all CV-MSCs were mentioned at passages 3-6.

The flow cytometry (with obtained antibodies from CA, San Diego, eBioscience, containing CD105, CD34, CD73, CD44, CD90, CD45, HLA-DR and IG1) was applied to detect the expression of cellular markers in CV-MSCs (passage 3). All of these markers are made up of both positive markers (CD90, CD73, CD44 and CD105) and negative ones (CD45, CD146, CD34, HLA-DR and IG1).

The medium was replaced with DMEM/F12 (Gibco, Carlsbad, CA) lacking FBS and the cells were cultured for another $24 \mathrm{~h}$, after CV-MSCs isolated from the placentas reached $80 \%$ confluence. What's more, the medium went through a filtration through a filter $(0.22 \mu \mathrm{m})$ (Millipore, Billerica, MA) after a twelve-minute centrifugation $(1,200 \times \mathrm{g})$. And the medium was used for later experiments. Besides, as control $\mathrm{CM}$, the DMEM/F12 medium lacking FBS were adopted.

From the Type Culture Collection China Centre, HUVEC were purchased. P1 cells were human umbilical vein endothelial cells. To culture two cells in an incubator with $37^{\circ} \mathrm{C}$ and $5 \% \mathrm{CO} 2, \mathrm{DMEM} / \mathrm{F} 12$ including $10 \%$ FBS was adopted.

\section{Transient transfection, lentivirus infection}

Transient transfections for lentivirus infection were performed by Lipofectamine 2000 (Invitrogen, Carlsbad, USA). In order to set uo stable transfected cell lines, the transfected cells were isolated as single clones after puromycin treatment.

The sequences of the shRNAs used are the following: sh-Control, 5'- GCAAGCTGACCCTGAAGTT -3'; shABCA9, 5'-GGAATGGACTTTGTGTCTT-3'.

Control lentivirus (Vector), lentiviral constructs expressing full-length ABCA9 were used to establish stably overexpressed cell lines.

\section{Western blot and quantitative real-time PCR (qRT-PCR)}

qRT-PCR and western blot were act as previously described ${ }^{23}$. As for western blot, the primary antibodies were applied: ABCA9 (ab192386, Abcam, Cambridge, USA; 1:1000), p-AKT (ser473, \#4060, CST; 1:1000), AKT (\#4685, CST; 1:1000), p-GSK3ß (ser9, \#5558, CST; 1:1000), $\beta$-catenin (\#8480, CST; 1:1000), GAPDH (ab181603, Abcam; 1:5000). Previously, they were used in the following sequence: ABCA9, forward, 5'- 
TCCGGGTAAGGGGAGTCAAT-3', reverse, 5'-GCCAAGGTCTTTCACACAACT-3'; GAPDH forward, 5'AATGGGCAGCCGTTAGGAAA-3', reverse, 5'- GCGCCCAATACGACCAAATC-3'.

\section{Cell Proliferation Analysis}

In the 96 -well plates, cells $\left(5 \times 10^{3} /\right.$ well) were embedded. According to the manufacturer's instructions, the CCK-8 reagent (Thermo Fisher Scientific) was applied to examine cell proliferation every day. All samples were tested at $450 \mathrm{~nm}$ with a microplate reader. Through three independent experiments, the growth curves were justified.

Tube formation assay

\section{Immunofluorescence analysis}

Under the condition of a density of per well for $48 \mathrm{~h}$, cells $\left(5 \times 10^{4} /\right.$ well $)$ were embeded on coverslips and later incubated with a primary antibody CD31 (ab134168, Abcam, Cambridge, USA; 1:100) or a-SMA (ab5831, Abcam; 1:100). Then, the coverslips were incubated with fluoresceinisothiocyanate (FITC)conjugated or rhodamine-conjugated goat antibodies against mouse or rabbit IgG (anti-rabbit IgG, Abcam; anti-mouse IgG, Abcam). Images were taken with a confocal laser-scanning microscope after counterstaining with DAPI.

\section{Immunohistochemistry}

In order to fix PE placenta $(n=20) 4 \%$ and the normal human term placenta $(n=20)$, paraformaldehyde was adopted for $60 \mathrm{~min}$. The tissues were embedded in paraffin, sliced into 4- $\mu \mathrm{m}$ sections, and then deparaffinized. Later, for antigen retrieval, the slides were boiled in 6.0-pH sodium citrate buffer (10 mM) for 7 min under one hundred and twenty degrees Celsius. At the same time, Hydrogen peroxide was adopted to block endogenous peroxidase for $10 \mathrm{~min}$. Each of the slides was washe d with TBS three times for 5 min. The TBS contained 0.05\% Tween 20 (TBS/T; Merck; Darmstadt, Germany). Next, these slides were incubated with anti-P62 antibodies $(1: 1,000)$ and monoclonal anti-STAT3 antibodies (1:200) for $12 \mathrm{~h}$ under four degrees Celsius. Moreover, under thirty-seven degrees Celsius, diluted biotinylated secondary antibodies were applied to incubate the sections for $20 \mathrm{~min}$. The target proteins were visualized by fresh DAB solution; later, haematoxylin was used as a tissue counterstain. The expression of the target proteins was independently assessed by two observers with an optical microscope (Olympus FV500, Tokyo, Japan). The area was analyzed via image-Pro Plus 5.1, with the intensity of staining in five random regions (200x magnification); by doing this, the expression level of proteins was evaluated.

\section{Colony forming assay}

A total of 500 - 1000 HUVECs were incubated in 6-well plates for 14 days. Paraformaldehyde was then used to fix the colonies which were afterwards stained with a crystal violet solution for visualization.

\section{RNA sequencing}


Samples were collected using TRIzol ( $1 \mathrm{ml}$ ) (Thermo Fisher Scientific), and were placed at minus $80^{\circ} \mathrm{C}$. According to the instructions for an Illumina TruSeq RNA Sample Prep Kit, the libraries were prepared, and on a MiSeq instrument, the sequencing was conducted. Analysis of data (from Annoroad Gene Technology, Beijing, China) was performed on RSEM software. With labeling of no. SRR11493647-no. SRR11493652, all RNA-seq data are available at Sequence Read Archive (SRA).

\section{Tube formation assay}

Tube formation assays were conducted according to previous description ${ }^{24}$. Matrigel was added to the pre-chilled culture plates (Corning; Midland, Michigan, USA) and they were put under $37^{\circ} \mathrm{C}$ for $30 \mathrm{~min}$. Then with different treatments, $2 \times 10^{4}$ HUVEC cells were embedded in each well and incubated for $6 \mathrm{~h}$. Furthermore, formation was evaluated and photographed by a light microscope.

\section{Matrigel Plug Assay}

Five-week-old male BALB/c mice were applied for the Matrigel plug assay. Those mice were purchased from the Shanghai Animal Centre (Shanghai, China). All procedures on them were conducted after being permitted by the Animal Care \& Use Committee of the Affiliated Hospital of Qingdao University, China. 500 $\mu$ of Matrigel containing 20 units of heparin, ABCA9 overexpressed HUVECs or vector transfected were injected subcutaneously into the ventral area of the mice. The mice died and the Matrigel plugs were spread from the skin and photographed after $7 \mathrm{~d}^{25}$.

\section{Statistical Analysis}

Statistical analyses were conducted with the use of unpaired two-tailed Student's $t$-test. And Data are given as mean \pm SEM. All experiments were conducted with more than three replicates. To analyze those data, GraphPad Prism version 7.00 software program (GraphPad; La Jolla, USA) was adopted. After taking a $P$ value $<0.05$ into consideration, a significant difference was worked out.

\section{Results}

\section{CV-MSCs promote the proliferation and tube formation of HUVEC cells in vitro via ABCA9}

First, we isolated CV-MSCs from healthy placentas, and then, the CCK-8, tube formation assays and colony forming assays was used to figure out the angiogenesis and proliferation of HUVEC dealt with or without CV-MSC conditional medium (CM). The HUVEC treated with CV-MSC CM displayed significantly higher proliferation rates and angiogenesis abilities than those untreated cells (Fig. $1 \mathrm{a}-\mathrm{C}$, all $\mathrm{P}<0.01$ ). To further elucidate the possible molecular mechanisms of CV-MSCs CM, differentially expressed genes (DEGs) in CM-treated HUVECs were investigated using RNA sequencing, and the molecular mechanism of underlying functional changes in HUVEC was explored. We compared the transcriptomes between HUVEC cells co-cultured with or without CV-MSCs CM for $48 \mathrm{~h}$, and 4006 upregulated and 4412 downregulated genes were found to change more than two-fold (Fig. 1 d). Based on the results, ABCA9 was found to be 
downregulated significantly in HUVECs treated with CV-MSCs CM. The result was clarified by western blot and qRT-PCR assays (Fig. 1e-f).

These outcomes showed the CV-MSCs promoted HUVEC proliferation and angiogenesis, and ABCA9 was upregulated in this process.

\section{$A B C A 9$ is downregulated in the placenta of PE pregnancies}

To better understand the role ABCA9 played in placenta of PE patients, we examined the protein and mRNA expression levels in placenta from normal and PE pregnancies by immunohistochemistry, western blot and qRT-PCR. The immunohistochemistry assays reveal a lower expression of ABCA9 in PE placenta (Fig. 2a). Our qRT-PCR results confirmed that mRNA levels of ABCA9 were significant decreased in PE placenta (Fig. 2b). In consistence with the changes of mRNA levels, the ABCA9 protein levels were also significantly decreased in PE placenta (Fig. 2C). Our results suggested that ABCA9 could be inhibited in placenta by PE pathological process, which suggested that $A B C A 9$ could play an role in the pathophysiological process of PE.

\section{ABCA9 promotes HUVEC proliferation and angiogenesis}

To better understand the role ABCA9 played in HUVEC after CV-MSC CM treatment, HUVECs were transfected with ABCA9 siRNA and overexpression lentiviruses. The mRNA levels of ABCA9 were examined, and significant decrease and increase of ABCA9 mRNA levels were respectively observed in transfected HUVECs (Fig. 3a). In consistence with the changes of mRNA levels, the ABCA9 protein levels were also significantly changed in transfected HUVECs compared to the control cells (Fig. 3b). Moreover, the growth of HUVECs was significantly inhibited in the presence of ABCA9 siRNA, while the ABCA9 overexpression promoted HUVECs proliferation (Fig. 3C). In addition, we examined the colony and tube formation of transfected HUVECs, and found that the cellular colony formation and angiogenesis changes in the corresponding HUVECs shared the same curve as observed changes in proliferation (Fig. $2 \mathrm{~d}-\mathrm{e})$. Our results suggested that ABCA9 could be promoted by CV-MSCs which regulated the proliferation and angiogenesis of HUVECs.

\section{ABCA9 enhances cell proliferation and angiogenesis through AKT pathway in vivo and in vitro.}

In a phosphatidylinositol-3 kinase-dependent manner, Akt is a serine/threonine protein kinase activated by cytokines and various growth factors ${ }^{26}$. It is well known that Akt signaling pathways regulates vascular homeostasis and angiogenesis through antiapoptotic activity in endothelial cells ${ }^{27,28}$, suggesting that it is involved in $A B C A 9$ associated HUVEC regulation. To understand whether the akt signaling plays a part in promoting effects of ABCA9, protein levels of $\beta$-catenin, $p$-GSK3 $\beta$, p-akt and akt in HUVEC treated with ABCA9 siRNA and primary cultured human umbilical vein endothelial cells treated with $A B C A 9$ overexpressed plasmids were examined by western blot (Fig. $2 \mathrm{~g}$ ). Our results confirmed that the Akt signaling pathways could be regulated by ABCA9 in HUVECs. 
The ABCA9 overexpressed HUVECs were treated with or without the PI3K-Akt pathway specific inhibitor LY294002 (ab120243) in order to justify the role of akt signaling played in HUVECs proliferation and angiogenesis regulation of ABCA9 (Abcam Biotech, Shanghai, China). Therefore, the AKT and p-AKT expressions were examined with the help of western blot in ABCA9 overexpression primary cultured human umbilical vein endothelial cells with or without LY294002. The relative p-AKT expression levels were significantly upregulated in the ABCA9 overexpressed HUVECs, and LY294002 could significantly hinder the elevated p-AKT expression (Fig. 4a). Moreover, the CCK-8 assays revealed that the ABCA9 overexpression markedly promoted cell proliferation, which could be attenuated by LY294002 (Fig. 4b). As our expected, $A B C A 9$ overexpression resulted in the promotion of tube and colony formation of primary HUVECs, and impaired angiogenesis and colony formation ability was also observed in ABCA9 overexpressed HUVECs treated with LY294002 (Fig. 4c-d).

The results suggested the possible involvement of ABCA9 in modulating the AKT pathway in CV-MSCs treated HUVEC. Then, we investigated if the CV-MSCs could regulate the proliferation and angiogenesis of HUVECs through ABCA9 regulation in vitro.

To further confirm whether akt signaling plays a role in ABCA9 mediated HUVEC promoting effect, we then investigated the angiogenesis and proliferation of HUVECs by Metrigel plug angiogenesis assay (Fig. 4e). HUVECs with ABCA9 overexpression showed faster proliferation and angiogenesis, and LY294002 could attenuate the effect of ABCA9 overexpression.

To summarize, these data indicate that CV-MSC-CM may activate the ABCA9-AKT pathway to promote the proliferation and angiogenesis of HUVECs.

\section{Discussion}

Preeclampsia and their complications during pregnancy are main causes of maternal morbidity and mortality, and endothelial cell dysfunction has been shown to be a central event in this pathophysiology ${ }^{29}$. When endothelium is influenced by oxidation and inflammation, developing dysfunction includes increased cell adhesion, losing the vasodilation and vasoconstriction balance ${ }^{30,31}$. Due to aberrant placentation and shallow invasion of trophoblast cells in PE, multiple high levels of inflammatory cytokines were released, leading to excessive vascular endothelium injury ${ }^{32}$. Many researches have demonstrated that MSCs could secrete greater amounts of chemokines and various growth factors, and the therapeutic potential of MSCs can be applied to many diseases ${ }^{33}$. Despite limited information on MSCs regulating functional development in placentas, several studies indicate that MSCs could protect endothelial cells from injury ${ }^{34}$.

Human Mesenchymal Stem Cells (hMSCs) are multipotent stem cells that are able to renew themselves and grow into tissues with different functions ${ }^{35}$. In vivo hMSCs are sources of trophic factors which are capable of inducing intrinsic stem cells to fix damaged tissues and modulating the immune system ${ }^{36}$. For the present, a great number of clinical trials are applying hMSCs as therapeutic purposes in many 
immune diseases, and the results are promising ${ }^{37}$. The placenta is made up of villous and smooth chorion, the amnion, decidua basalis, umbilical cord and chorionic plate ${ }^{38}$. The chorionic plate has already been an available source of MSCs, which exhibiting undifferentiation marker genes expression and transdifferentiation, such as TERT, OCT3/4, KLF4, SOX2 and c-MYC ${ }^{39}$. The placenta is a readily reliable and available source of allogeneic cells apart from other sources such as cartilage, bone marrow, ligament and adipose tissue in various cases of genetic and degenerative diseases ${ }^{40}$. As for industrial scale-up for a sustainable supply of a large amount of quality-controlled and affordable cells, chorionic villous mesenchymal stem cells isolation and subsequent propagation are suitable $e^{41}$.

To understand the function of CV-MSCs in improving endothelial function, we performed the RNA-seq analysis in CV-MSCs treated HUVEC. Then, ABCA9 was found to be significantly increased in CV-MSC CM treated HUVECs, which also shows lower expression in placental tissue from PE pregnancies. Moreover, activation of Akt signaling induced by ABCA9 overexpression may contribute to the increased angiogenesis and proliferation of HUVEC mediated by CV-MSCs.

The A-subclass of ATP-binding cassette $(A B C)$ transporters is a well-protected family member of potent lipid transporters ${ }^{42}$. The roles of the ABCA6-like subgroup transporters are still unknown ${ }^{43}$. And they have been identified as a unique gene cluster on human chromosome 17q24. Several research revealed that ABCA9 mRNAs could be isolated from vascular endothelial cells and placenta. This is possible to indicate the importance of $A B C A 9$ in regulating vascular endothelial cells function ${ }^{43-45}$. Our results confirmed that ABCA9 could be regulated by CV-MSC and take part in the vascular endothelial cell proliferation promoting effects induced by CV-MSCs. ABCA9 overexpression in HUVEC significantly increased the proliferation and angiogenesis with an activation of AKT signaling.

Many studies have confirmed that mesenchymal stem cells could enhance endothelial cells function through AKT activation ${ }^{46}$. AKT and Wnt signaling pathways are of great significance for the survival and angiogenesis of cells through modulating endothelial cell migration, proliferation, remodeling, vascular system maturation and vascular sprouting ${ }^{47-49}$. In this study, the elevated protein levels of $p$-GSK3 $\beta$ and p-AKT are probably associated with the increased cell proliferation in ABCA9 overexpressed HUVECs, and the outcome is consistent with previous findings in many functional endothelial cells $s^{50}$.

In summary, it is revealed in our study that ABCA9 activation induced by CV-MSCs promoted the HUVEC proliferation and angiogenesis in vitro, partially due to activation of AKT pathways in HUVECs. However, whether CV-MSCs affect other placenta-based cells was still unknown; therefore, further studies are needed on whether CV-MSCs have similar effects on them. Further studies should be performed to understand to how $A B C A 9$ regulates AKT pathways, which will be important for a thorough understanding of the $P E$ vessel pathogenesis.

\section{Declarations}


The study was approved by the Ethics Committee of the Qingdao University Affiliated Hospital. All participants provided a written informed consent.

\section{Consent for publication}

Not applicable.

\section{Availability of supporting data}

The datasets used and/or analyzed during the current study are available from the corresponding author on reasonable request.

\section{Competing interests}

The authors declare no potential conflicts of interest.

\section{Funding}

This study was supported by Postdoctoral Science Foundation of China (2019M652329), the Clinical Medicine+X Project of the Affiliated Hospital of Qingdao University.

\section{Authors' contributions}

Conceived and designed the experiments: YC, YW and WP. Performed the experiments: JZ, XH, YZ, GG and JZ. Analyzed the data: RH, CL, HZ and ML. Wrote the paper: YC, JZ and YC. All authors read and approved the final manuscript.

\section{Acknowledgements}

No funders had no role in the study design, in the collection, analysis, and interpretation of the data or in writing the manuscript. We thank the Department of Obstetrics, the Affiliated Hospital of Qingdao University, Qingdao, China, for providing tissue samples.

\section{References}

1 Al-Ofi, E. A. \& Anumba, D. O. Ligands of toll-like receptors 2/4 differentially alter markers of inflammation, adhesion and angiogenesis by monocytes from women with pre-eclampsia in co-culture with endothelial cells. J Reprod Immuno/121, 26-33, doi:10.1016/j.jri.2017.05.002 (2017).

2 Gao, Q. et al. Upregulation of P53 promoted G1 arrest and apoptosis in human umbilical cord vein endothelial cells from preeclampsia. J Hypertens34, 1380-1388, doi:10.1097/HJH.0000000000000944 (2016).

3 Yetik-Anacak, G. \& Catravas, J. D. Nitric oxide and the endothelium: history and impact on cardiovascular disease. Vascul Pharmaco/45, 268-276, doi:10.1016/j.vph.2006.08.002 (2006). 
4 Konukoglu, D. \& Uzun, H. Endothelial Dysfunction and Hypertension. Advances in experimental medicine and biology956, 511-540, doi:10.1007/5584_2016_90 (2017).

5 Brown, D. Preeclampsia, hypertension, and a possible treatment for preeclampsia. Med Hypotheses140, 109669, doi:10.1016/j.mehy.2020.109669 (2020).

6 Amaral, L. M., Cunningham, M. W., Jr., Cornelius, D. C. \& LaMarca, B. Preeclampsia: Iong-term consequences for vascular health. Vasc Health Risk Manag11, 403-415, doi:10.2147/VHRM.S64798 (2015).

7 Lip, S. V. et al. Early-onset preeclampsia, plasma microRNAs, and endothelial cell function. Am J Obstet Gyneco/222, 497 e491-497 e412, doi:10.1016/j.ajog.2019.11.1286 (2020).

8 Karatza, A. A. \& Dimitriou, G. Preeclampsia emerging as a novel risk factor for cardiovascular disease in the offspring. Curr Pediatr Rev, doi:10.2174/1573396316666191224092405 (2019).

9 Ding, D. C., Shyu, W. C. \& Lin, S. Z. Mesenchymal stem cells. Cell transplantation20, 5-14, doi:10.3727/096368910X (2011).

10 Samsonraj, R. M. et al. Concise Review: Multifaceted Characterization of Human Mesenchymal Stem Cells for Use in Regenerative Medicine. Stem cells translational medicine6, 2173-2185, doi:10.1002/sctm.17-0129 (2017).

11 Lv, F. J., Tuan, R. S., Cheung, K. M. \& Leung, V. Y. Concise review: the surface markers and identity of human mesenchymal stem cells. Stem cells32, 1408-1419, doi:10.1002/stem.1681 (2014).

12 Fu, X. et al. Mesenchymal Stem Cell Migration and Tissue Repair. Cells8, doi:10.3390/cells8080784 (2019).

13 Keshtkar, S., Azarpira, N. \& Ghahremani, M. H. Mesenchymal stem cell-derived extracellular vesicles: novel frontiers in regenerative medicine. Stem cell research \& therapy9, 63, doi:10.1186/s13287-0180791-7 (2018).

14 Anasiz, Y., Ozgul, R. K. \& Uckan-Cetinkaya, D. A New Chapter for Mesenchymal Stem Cells: Decellularized Extracellular Matrices. Stem Cell Rev Rep13, 587-597, doi:10.1007/s12015-017-9757-x (2017).

15 Ceserani, V. et al. Angiogenic and anti-inflammatory properties of micro-fragmented fat tissue and its derived mesenchymal stromal cells. Vasc Cel/8, 3, doi:10.1186/s13221-016-0037-3 (2016).

16 Babajani, A., Soltani, P., Jamshidi, E., Farjoo, M. H. \& Niknejad, H. Recent Advances on Drug-Loaded Mesenchymal Stem Cells With Anti-neoplastic Agents for Targeted Treatment of Cancer. Front Bioeng Biotechno/8, 748, doi:10.3389/fbioe.2020.00748 (2020). 
17 Carvalho, A. É. S., Sousa, M. R. R., Alencar-Silva, T., Carvalho, J. L. \& Saldanha-Araujo, F. Mesenchymal stem cells immunomodulation: The road to IFN-y licensing and the path ahead. Cytokine \& growth factor reviews47, 32-42, doi:10.1016/j.cytogfr.2019.05.006 (2019).

18 Meirelles Lda, S., Fontes, A. M., Covas, D. T. \& Caplan, A. I. Mechanisms involved in the therapeutic properties of mesenchymal stem cells. Cytokine \& growth factor reviews20, 419-427, doi:10.1016/j.cytogfr.2009.10.002 (2009).

19 Wang, Z. et al. Transplantation of human villous trophoblasts preserves cardiac function in mice with acute myocardial infarction. Journal of cellular and molecular medicine21, 2432-2440, doi:10.1111/jcmm.13165 (2017).

20 Hsuan, Y. C., Lin, C. H., Chang, C. P. \& Lin, M. T. Mesenchymal stem cell-based treatments for stroke, neural trauma, and heat stroke. Brain Behav6, e00526, doi:10.1002/brb3.526 (2016).

21 Ding, H. et al. Transplantation of placenta-derived mesenchymal stem cells reduces hypoxic-ischemic brain damage in rats by ameliorating the inflammatory response. Cell Mol Immuno/14, 693-701, doi:10.1038/cmi.2015.99 (2017).

22 Cunningham, C. J., Redondo-Castro, E. \& Allan, S. M. The therapeutic potential of the mesenchymal stem cell secretome in ischaemic stroke. J Cereb Blood Flow Metab38, 1276-1292, doi:10.1177/0271678X18776802 (2018).

$23 \mathrm{Chu}, \mathrm{Y}$. et al. Human omental adipose-derived mesenchymal stem cells enhance autophagy in ovarian carcinoma cells through the STAT3 signalling pathway. Cellular signalling69, 109549, doi:10.1016/j.cellsig.2020.109549 (2020).

24 Gentile, M. T., Pastorino, O., Bifulco, M. \& Colucci-D'Amato, L. HUVEC Tube-formation Assay to Evaluate the Impact of Natural Products on Angiogenesis. Journal of visualized experiments : JoVE, doi:10.3791/58591 (2019).

$25 \mathrm{Heo}, \mathrm{S}$. H. \& Cho, J. Y. ELK3 suppresses angiogenesis by inhibiting the transcriptional activity of ETS-1 on MT1-MMP. Int J Biol Sci10, 438-447, doi:10.7150/ijbs.8095 (2014).

26 Yu, H., Littlewood, T. \& Bennett, M. Akt isoforms in vascular disease. Vascul Pharmacol71, 57-64, doi:10.1016/j.vph.2015.03.003 (2015).

27 Song, M. \& Finley, S. D. ERK and Akt exhibit distinct signaling responses following stimulation by proangiogenic factors. Cell communication and signaling : CCS18, 114, doi:10.1186/s12964-020-00595-w (2020).

28 Shiojima, I. \& Walsh, K. Role of Akt signaling in vascular homeostasis and angiogenesis. Circulation research90, 1243-1250, doi:10.1161/01.res.0000022200.71892.9f (2002). 
29 Sandvoss, M., Potthast, A. B., von Versen-Hoynck, F. \& Das, A. M. HELLP Syndrome. Reprod Sci24, 568574, doi:10.1177/1933719116667216 (2017).

30 van Rijn, B. B. et al. Postpartum Circulating Markers of Inflammation and the Systemic Acute-Phase Response After Early-Onset Preeclampsia. Hypertension67, 404-414, doi:10.1161/HYPERTENSIONAHA.115.06455 (2016).

31 Pecher, S. J., Potthast, A. B., von Versen-Hoynck, F. \& Das, A. M. Impact of Short-Term Hypoxia on Sirtuins as Regulatory Elements in HUVECs. J Clin Med9, doi:10.3390/jcm9082604 (2020).

32 Li, M., Qian, L., Yu, J. \& Zou, Y. Interleukin-35 inhibits human umbilical vein endothelial cell injury induced by sera from pre-eclampsia patients by up-regulating S100A8 protein expression. Hypertens Pregnancy39, 126-138, doi:10.1080/10641955.2020.1744000 (2020).

33 Abumaree, M. H. et al. Preconditioning human natural killer cells with chorionic villous mesenchymal stem cells stimulates their expression of inflammatory and anti-tumor molecules. Stem cell research \& therapy10, doi:10.1186/s13287-019-1153-9 (2019).

34 Basmaeil, Y. S. et al. Human chorionic villous mesenchymal stem/stromal cells protect endothelial cells from injury induced by high level of glucose. Stem cell research \& therapy 9 , doi:10.1186/s13287018-0984-0 (2018).

35 MacDonald, E. S. \& Barrett, J. G. The Potential of Mesenchymal Stem Cells to Treat Systemic Inflammation in Horses. Front Vet Sci6, 507, doi:10.3389/fvets.2019.00507 (2019).

36 Rodriguez-Fuentes, D. E. et al. Mesenchymal Stem Cells Current Clinical Applications: A Systematic Review. Archives of medical research, doi:10.1016/j.arcmed.2020.08.006 (2020).

$37 \mathrm{Du}, \mathrm{W}$. et al. VCAM-1+ placenta chorionic villi-derived mesenchymal stem cells display potent proangiogenic activity. Stem cell research \& therapy7, 49, doi:10.1186/s13287-016-0297-0 (2016).

$38 \mathrm{Ma}$, J. et al. Comparative analysis of mesenchymal stem cells derived from amniotic membrane, umbilical cord, and chorionic plate under serum-free condition. Stem cell research \& therapy10, 19, doi:10.1186/s13287-018-1104-x (2019).

39 Kim, Y. H., Park, T. C., Lee, G. \& Shin, J. C. Gene expression pattern of human chorion-derived mesenchymal stem cells during adipogenic differentiation. Yonsei Med J53, 1036-1044, doi:10.3349/ymj.2012.53.5.1036 (2012).

40 Saleh, M. et al. Perspective of placenta derived mesenchymal stem cells in acute liver failure. Cell Biosci10, 71, doi:10.1186/s13578-020-00433-z (2020).

41 Evangelista, M., Soncini, M. \& Parolini, O. Placenta-derived stem cells: new hope for cell therapy? Cytotechnology58, 33-42, doi:10.1007/s10616-008-9162-z (2008). 
42 Rempel, S., Stanek, W. K. \& Slotboom, D. J. ECF-Type ATP-Binding Cassette Transporters. Annu Rev Biochem88, 551-576, doi:10.1146/annurev-biochem-013118-111705 (2019).

43 Tachikawa, M. et al. Gene expression of A6-like subgroup of ATP-binding cassette transporters in mouse brain parenchyma and microvessels. Anat Sci Int93, 456-463, doi:10.1007/s12565-018-0435-0 (2018).

44 Imperio, G. E. et al. Gestational age-dependent gene expression profiling of ATP-binding cassette transporters in the healthy human placenta. Journal of cellular and molecular medicine23, 610-618, doi:10.1111/jcmm.13966 (2019).

45 Tachikawa, M., Toki, H., Tomi, M. \& Hosoya, K. Gene expression profiles of ATP-binding cassette transporter A and C subfamilies in mouse retinal vascular endothelial cells. Microvasc Res75, 68-72, doi:10.1016/j.mvr.2007.05.002 (2008).

46 Zhang, X. et al. Coculture of mesenchymal stem cells and endothelial cells enhances host tissue integration and epidermis maturation through AKT activation in gelatin methacryloyl hydrogel-based skin model. Acta Biomater59, 317-326, doi:10.1016/j.actbio.2017.07.001 (2017).

47 Bing-Hua Jiang, L.-Z. L. AKT Signaling in Regulating Angiogenesis. Current Cancer Drug Targets8, 19 26 (2008).

48 Weng, L., Enomoto, A., Ishida-Takagishi, M., Asai, N. \& Takahashi, M. Girding for migratory cues: roles of the Akt substrate Girdin in cancer progression and angiogenesis. Cancer science101, 836-842, doi:10.1111/j.1349-7006.2010.01487.x (2010).

49 Karar, J. \& Maity, A. PI3K/AKT/mTOR Pathway in Angiogenesis. Front Mol Neurosci4, 51, doi:10.3389/fnmol.2011.00051 (2011).

50 Chen, Y. et al. Infiltrating mast cells promote renal cell carcinoma angiogenesis by modulating PI3K>AKT->GSK3beta-->AM signaling. Oncogene36, 2879-2888, doi:10.1038/onc.2016.442 (2017).

\section{Figures}




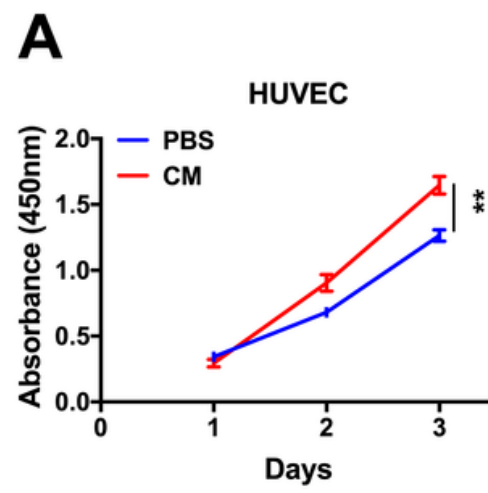

B

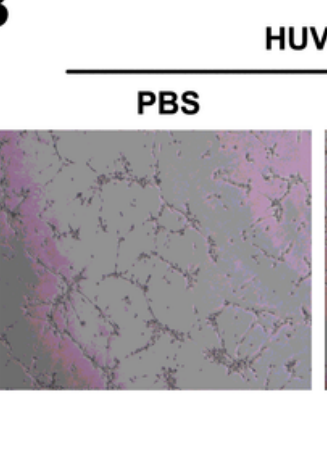

HUVEC

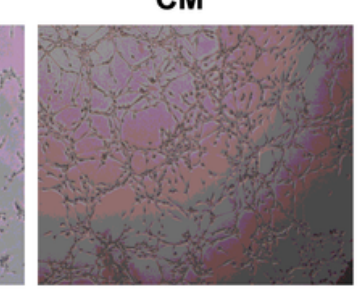

E

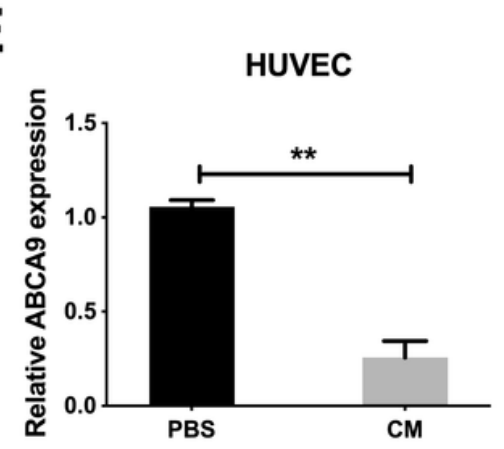

C

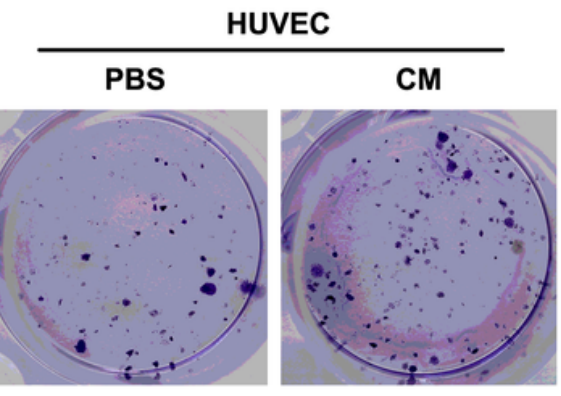

$\mathbf{F}$

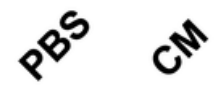

ABCA9

GAPDH

\section{Figure 1}

Fig1. CV-MSCs promote the proliferation and tube formation of HUVEC cells in vitro via ABCA9 (a) CCK8 assays from HUVEC cells co-cultured with PBS or CV-MSC CM to evaluate cell growth. (b) Colony forming assays from HUVEC cells co-cultured with PBS or CV-MSC CM. (c) Tube formation assay from HUVEC cells co-cultured with PBS or CV-MSC CM. (d) Volcano Plot of differential mRNA expression between PBS and from HUVEC cells co-cultured with PBS or CV-MSC CM groups from HUVEC cells. (e) qRT-PCR analysis of ABCA9 from PBS or from HUVEC cells co-cultured with PBS or CV-MSC CM groups. (f) Western blot analysis of ABCA9 from indicated cells. 

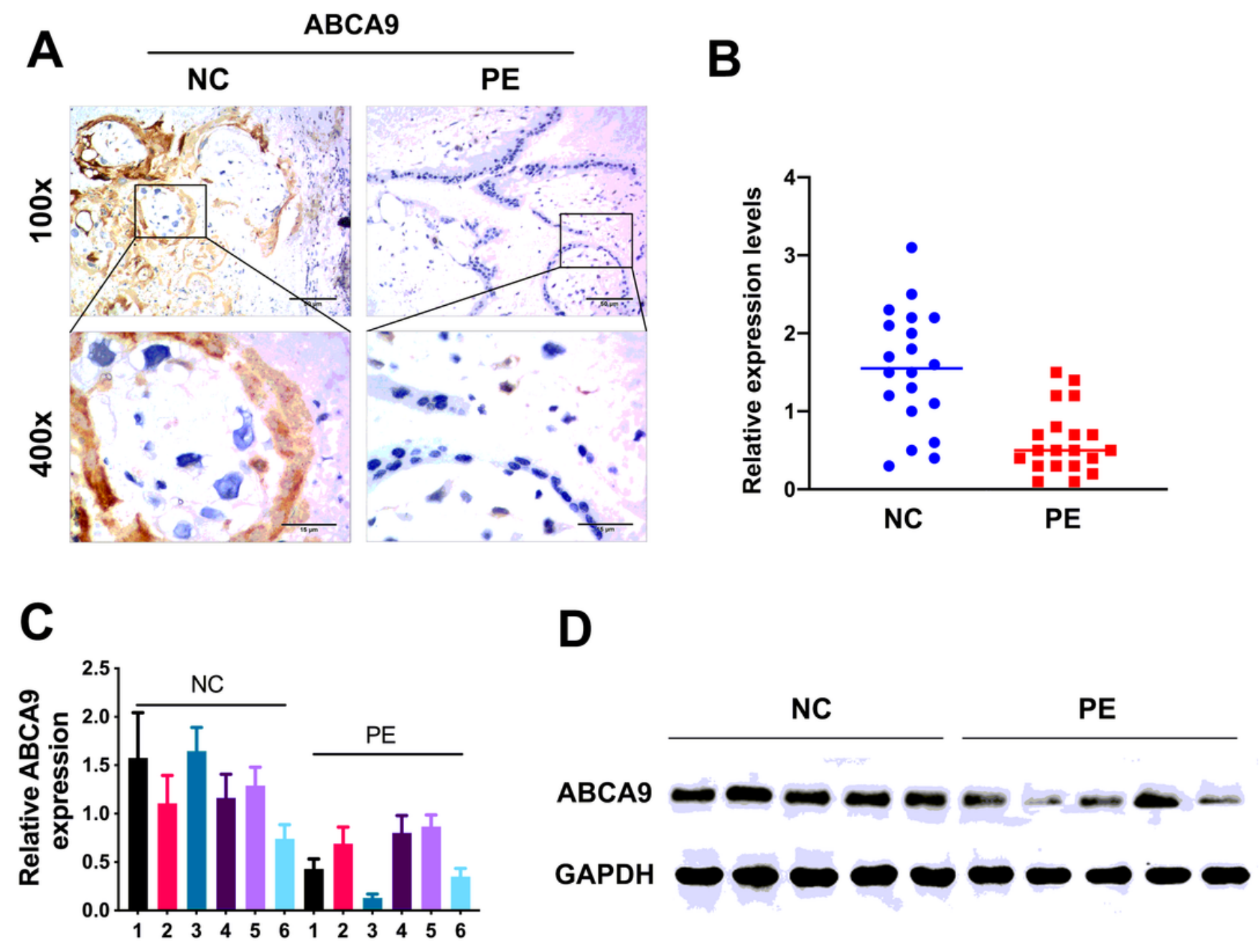

Figure 2

$A B C A 9$ is downregulated in the placenta of PE pregnancies. (a-b) Representative images of IHC staining of ABCA9 in normal control $(N C ; n=20)$ tissues and PE $(n=20)$ tissues, and a histogram of relative expression. (b) Relative ABCA9 mRNA levels of placenta tissues from pregnant women with PE $(n=6)$ or normal control pregnancy $(\mathrm{NC} ; \mathrm{n}=6)$ as detected by $(\mathrm{c})$ qRT-PCR and (d) Western blot. GAPDH was used for normalization. 


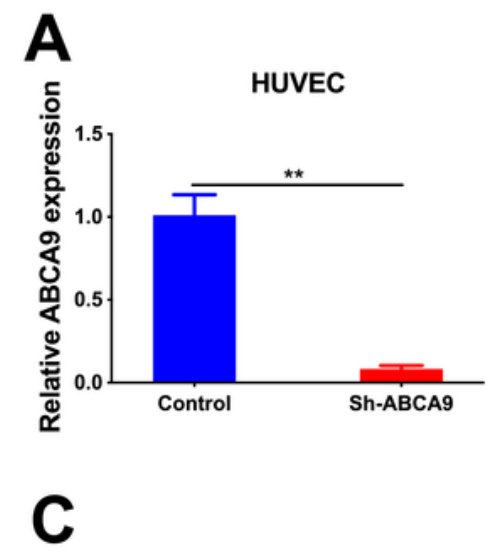

HUVEC

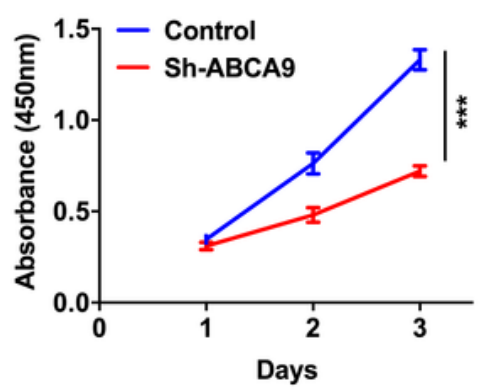

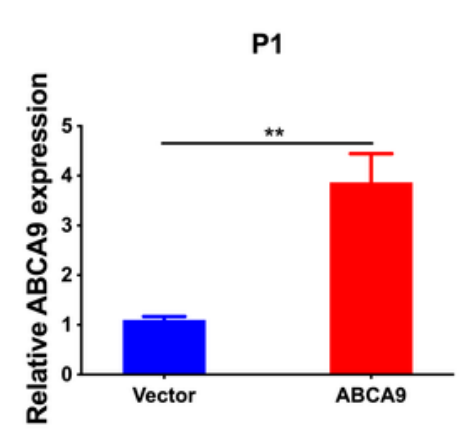

B

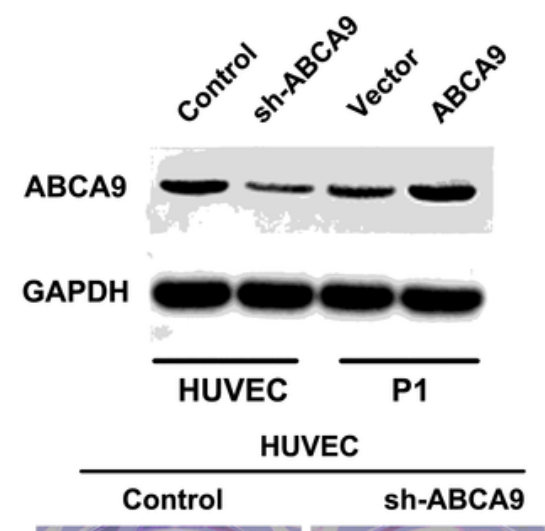

D

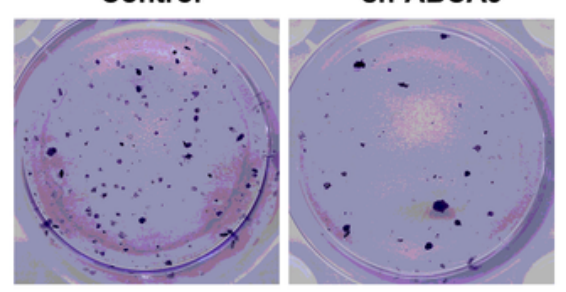

P1

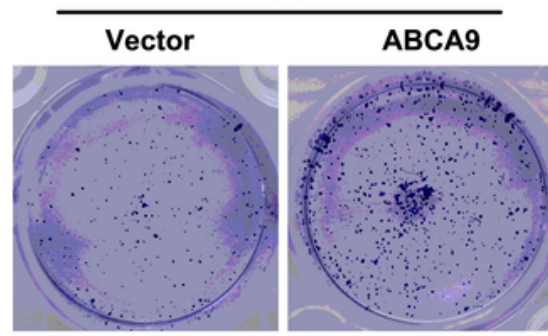

F

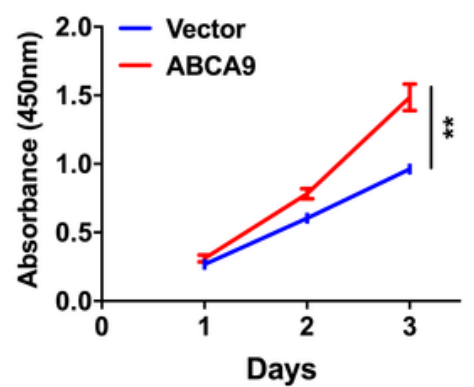

sh-ABCA9

E
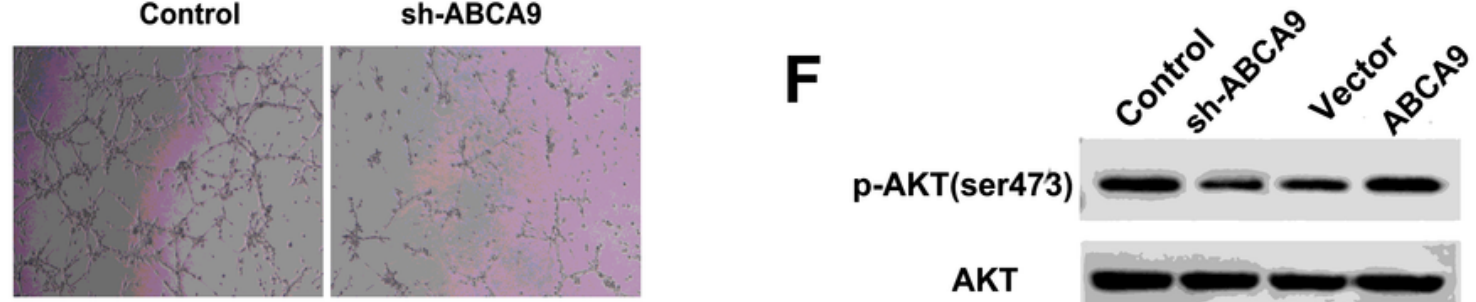

p-AKT(ser473)

AKT

p-GSK3 $\beta$ (ser9)

$\beta$-catenin

GAPDH
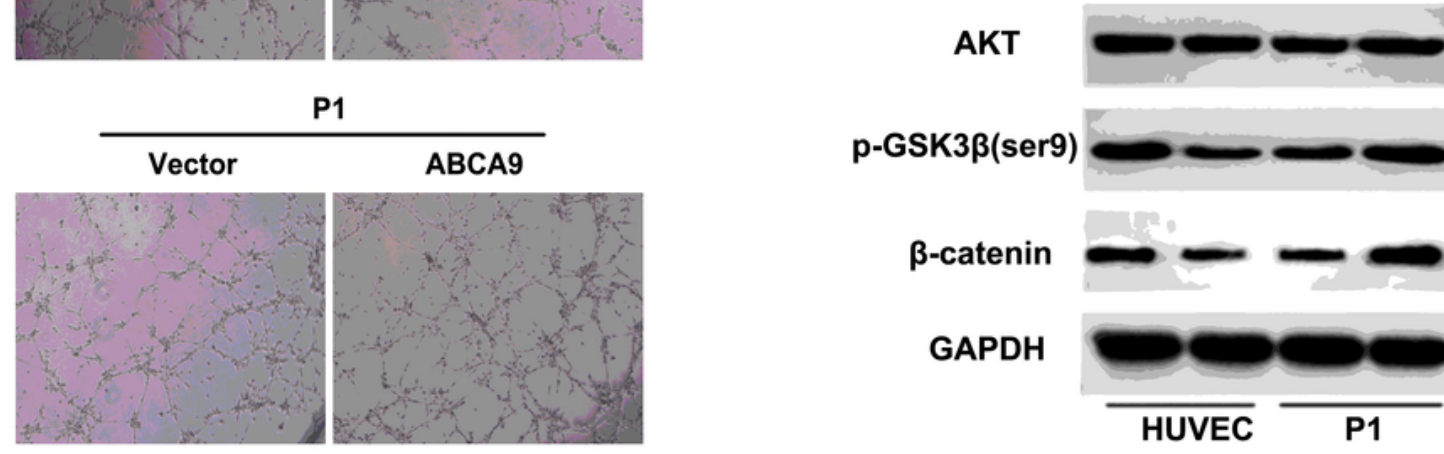

ABCA9

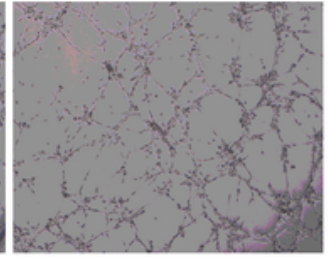


p-AKT(ser473), AKT, p-GSK3 $\beta$ (ser9) and $\beta$-catenin in stably transfected HUVEC and P1 cells. GAPDH was used for normalization.
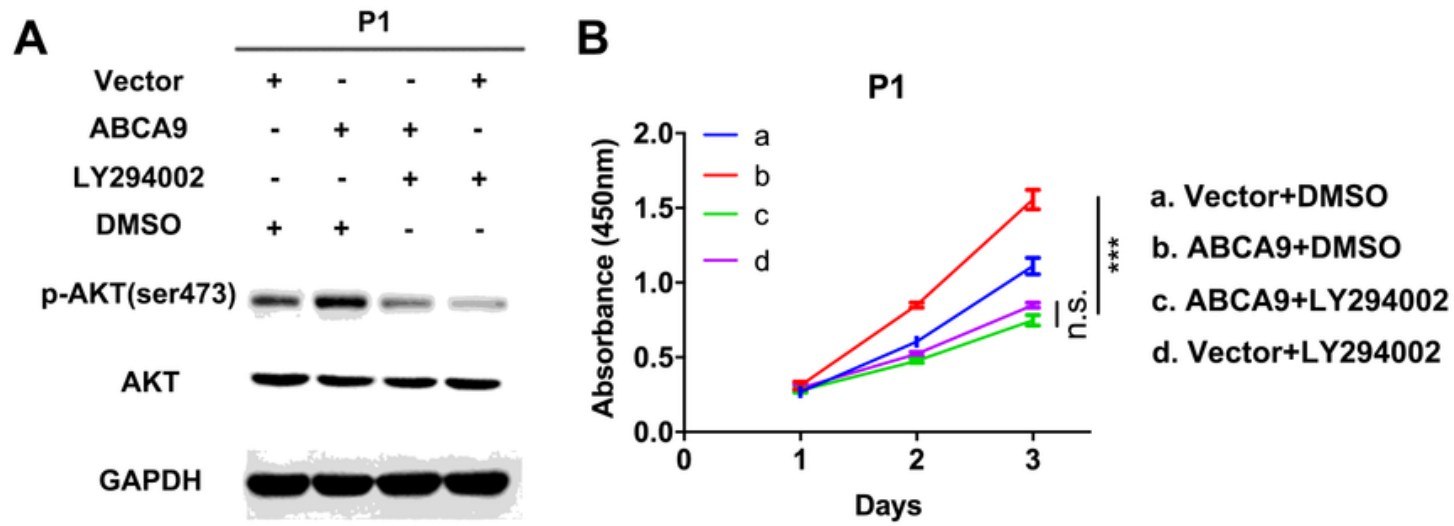

C

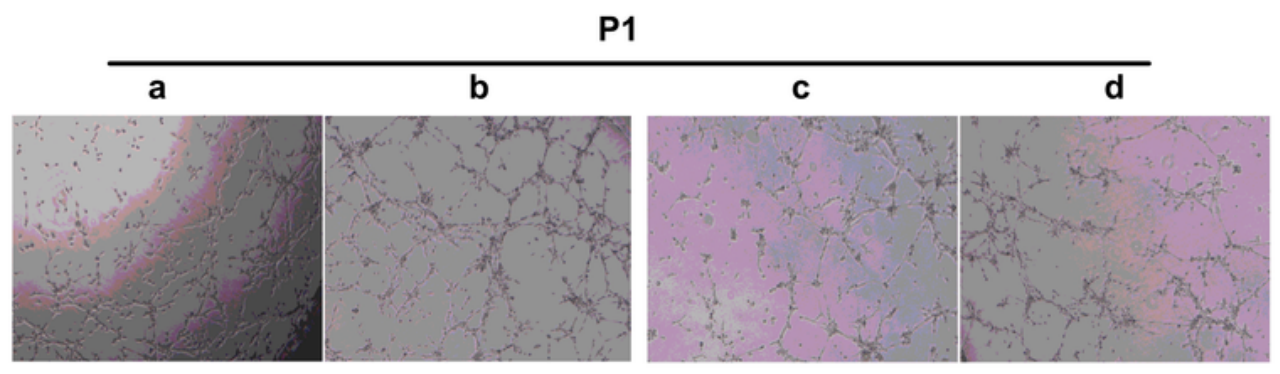

D

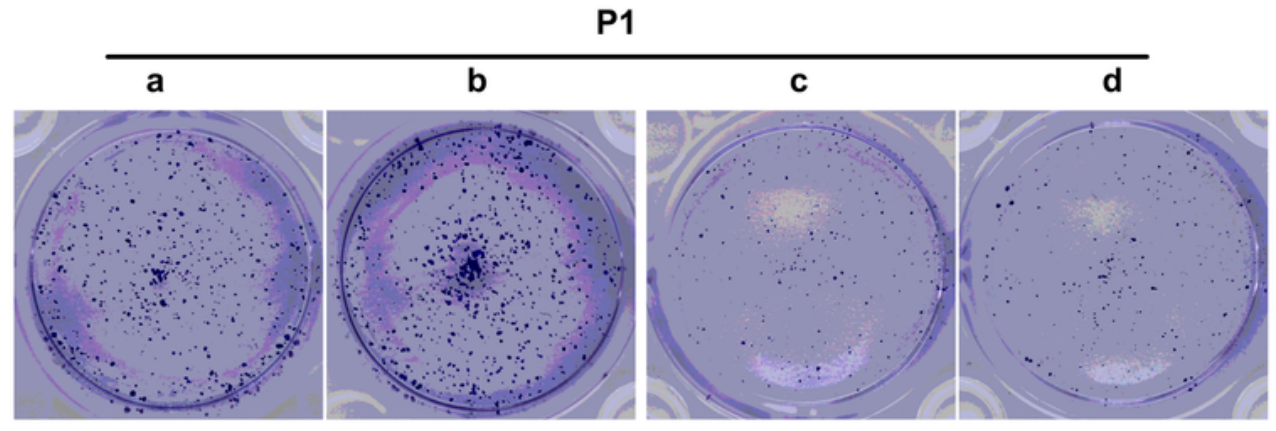

E P1

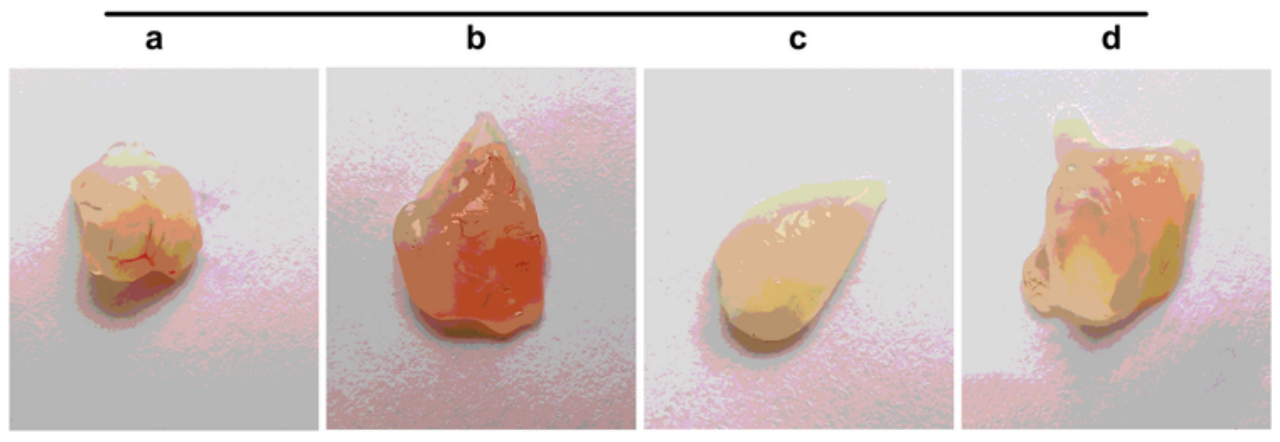

\section{Figure 4}

ABCA9 enhances cell proliferation and tube formation through AKT pathway in vivo and in vitro. (a) Western blot analysis of p-AKT(ser473), AKT in indicated stably transfected HUVEC and P1 cells treated

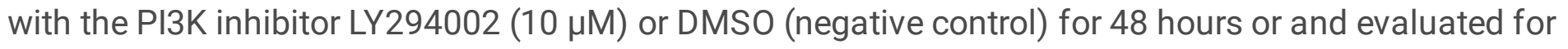


(b) Cell growth in the CCK8 assays and (c) Colony formation assays. (d) Tube formation assay in indicated group. (e) Representative images of Matrigel Plug Assay in indicated group. 